\title{
Diagnostic and therapeutic challenge - fibromyalgia
}

\section{Brygida Kwiatkowska}

Clinic of Early Arthritis, National Institute of Geriatrics, Rheumatology and Rehabilitation, Warsaw, Poland

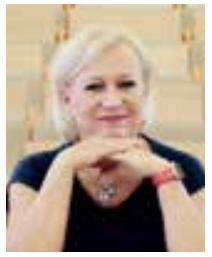

The first descriptions of fibromyalgia (FB) come from the nineteenth century, but it is still a disease that poses great diagnostic difficulties. This is due to the fact that biomarkers specific to the disease that could be used to diagnose it have not been detected so far.

Despite the existence of diagnostic criteria for FB, the mean time from the onset of the first symptoms to the diagnosis is 6.5 years. Most patients complain of chronic pain of the joints, muscles, head and the sacral area of the spine. These conditions are accompanied by fatigue, problems with falling asleep and cognitive deterioration. Often, patients also report stiffness, leg cramps, hypersensitivity to pressure, tingling and/or numbness, feelings of anxiety and depression, and facial pain [1]. A multitude of symptoms causes that the majority of patients are treated by specialists from various fields of medicine before they are directed to rheumatologist. Over $20 \%$ of patients is not able to work because of the severity of FB symptoms, and as many as $25 \%$ do not work full time for this reason [2].

Although the pathogenesis of FB remains not fully understood, a number of agents are considered as triggering factors. Among them are neuroendocrine and autonomic nervous system disorders, certain genetic factors, psychosocial factors and environmental stressors [3]. These factors are also involved in the pathophysiology of other disorders, which often coexist with FB, such as irritable bowel syndrome, disorders of the temporomandibular joint, depression and anxiety disorders.

Fibromyalgia is not a rare disease, as it occurs in $2-8 \%$ of the general population. It mainly affects women aged 20-55 [3]. It has also been shown that patients with FB family history have an increased risk of developing the illness even 8.5 fold.

Patients with FB symptoms have increased sensitivity to stimuli such as heat and cold, as well as to mechanical pressure and ischemia. There is evidence that FB is characterized by increased sensory activity mediated by mechanisms associated with the central nervous system similar to those found in neuropathic pain, where abnormal concentrations of serotonin and adrenaline - neurotransmitters of endogenous pain inhibiting pathways - are being observed [4].

It has been shown that in some patients the disease is the result of past infection (Epstein-Barr virus, viral hepatitis, Lyme disease) or persistent infection, as is the case with Herpes simplex virus infection.

Significant progress in our knowledge about new factors causing this disease, development of criteria and diagnostic methods - such as functional magnetic resonance of the brain - is not always sufficient to diagnose FB [5]. Therefore, this disease is still frustrating for both the doctor and the patient.

In most cases, the diagnosis requires many tests to exclude other causes of symptoms reported by the patient. On the other hand, each inflammatory rheumatic disease requires additional diagnostics for possible coexistence of FB. Coexistence of FB and inflammatory rheumatic disease hinders the proper monitoring of the latter, unless FB is taken into account. This can lead to a change or intensification of treatment of rheumatic disease without obtaining the expected efficacy of such treatment.

There are few drugs with documented efficacy in the treatment of FB. Many patients, despite the use of many combinations of therapeutic methods, do not achieve improvement and reduce pain. There are promising reports of a combination therapy of the antiviral drug famciclovir in combination with a non-steroidal antiinflammatory drug from the group of selective COX-2 inhibitors - celecoxib [6]. In the conducted studies, the effectiveness of such therapy has been shown to be higher than the treatment of chronic pain used so far. Such treatment is expected to affect latent herpes infection.

There is no doubt that FB is a major diagnostic and therapeutic challenge [7]. Dynamic expansion of knowledge about the pathogenesis of pain, inflammatory and 
behavioral processes, discoveries in the field of functional evaluation of the brain should make a recognition of FB faster and its treatment more effective.

The author declares no conflict of interest.

\section{References}

1. Theoharides TC, Tsilioni I, Arbetman L, et al. Fibromyalgia syndrome in need of effective treatments. J Pharmacol Exp Ther 2015; 355: 255-263.

2. Collado A, Gomez E, Coscolla R, et al. Work, family and social environment in patients with Fibromyalgia in Spain: an epidemiological study: EPIFFAC study. BMC Health Serv Res 2014; 14: 513 .
3. Wolfe F, Walitt B, Perrot S, et al. Fibromyalgia diagnosis and biased assessment: Sex, prevalence and bias. PLoS One 2018; 13: e0203755.

4. Bellato E, Marini E, Castoldi F, et al. Fibromyalgia syndrome: etiology, pathogenesis, diagnosis, and treatment. Pain Res Treat 2012; 2012: 426130.

5. Sluka KA, Clauw DJ. Neurobiology of fibromyalgia and chronic widespread pain. Neuroscience 2016; 338: 114-129.

6. Okifuji A, Gao J, Bokat C, Hare BD. Management of fibromyalgia syndrome in 2016. Pain Manag 2016; 6: 383-400.

7. Pridgen WL, Duffy C, Gendreau JF, Gendreau RM. A famciclovir + celecoxib combination treatment is safe and efficacious in the treatment of fibromyalgia. J Pain Res 2017; 10: 451-460.

The recognition of chronic pain syndromes are a major health concern is steadily growing.

We present in the coming study the linkage between diabetes and the fibromyalgia syndrome in a large database of the Israeli population with a review of the relevant medical literature.

Prof. Howard Amital Department of Medicine "B”, Sheba Medical Center, Tel-Hashomer, Israel

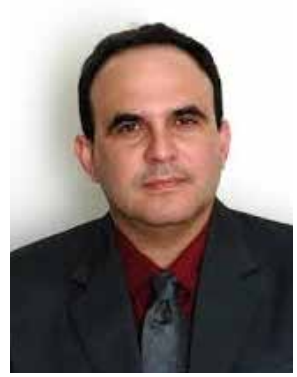

